\title{
THE EXPANSION PROBLEMS ASSOCIATED WITH REGULAR DIFFERENTIAL SYSTEMS OF THE SECOND ORDER*
}

\author{
BY
}

M. H. STONE

In 1908 Birkhoff defined a class of series generalizing the Fourier and Sturm-Liouville series which arise from ordinary linear homogeneous differential equations of the second order with linear homogeneous boundary conditions. He starts from a differential system of order $n$ with boundary conditions restricted to be regular and proves that an arbitrary function with a sufficient number of derivatives can be represented in terms of the solutions of this differential system. $\dagger$ Subsequently, Tamarkin extended the discussion to apply to functions integrable in the sense of Lebesgue by the device of comparing the formal Fourier and Birkhoff series for the same function. $\neq$ Recently the author has published further researches on the problem. $\S$

It is now proposed to complete in some respects our knowledge of regular differential systems of the second order. In our paper cited above, we left certain necessary conditions unstudied and now desire to fill this gap in the theory. $\|$ In addition, the attempt to discuss the formal expansions for functions integrable in accordance with definitions of integration more extended than that of Lebesgue has led us to results quite different from those hitherto established. We shall indicate the facts for functions integrable in the sense of Denjoy. The situation may be described roughly by saying that, whereas all the regular expansions associated with an arbitrary function integrable in the sense of Lebesgue have virtually the same behavior, the series formed for an arbitrary function integrable in the sense of Denjoy or non-absolutely integrable in the sense of Riemann fall into a non-denumerable infinity of distinct types exhibiting individual behavior. The result is due essentially to the fact that the Riemann-Lebesgue theorem does not hold true for nonabsolutely integrable functions. On the other hand, if the series for an arbi-

* Presented to the Society, October 30, 1926; received by the editors in December, 1926.

$\dagger$ Birkhoff, these Transactions, vol. 9 (1908), pp. 373-395, cited as $B_{1}$; and Rendiconti del Circolo Matematico di Palermo, vol. 36 (1913), pp. 115-126, cited as $\mathrm{B}_{2}$.

¥ Tamarkin, Rendiconti del Circolo Matematico di Palermo, vol. 34 (1910), pp. 345-382, cited as $\mathrm{T}_{1}$; also, On certain General Problems in the Theory of Ordinary Linear Differential Equations and the Expansion of an Arbitrary Function in Series, Petrograd, 1917, cited as $\mathrm{T}_{\mathbf{2}}$.

\& Stone, these Transactions, vol. 28 (1926), pp. 695-761, cited as S.

\| Compare the closing paragraph of S, §IV. 
trary function integrable in the sense of Denjoy are considered with regard to their summability by means of sufficiently high order, this difference in behavior is found to disappear.

\section{Preliminary lemmas}

We shall need a number of lemmas for our later work, and prefer to collect them in one spot for reference, though none of them is essentially new. In several places we refer to other papers for the proofs.

Lемма I. The differential equation

$$
u^{\prime \prime}+\left(\rho^{2}+g\right) u=0, \quad 0 \leqq x \leqq 1,
$$

where $\rho$ is a complex parameter and $g(x)$ an integral in the sense of Lebesgue, has two linearly independent solutions $u_{1}, u_{2}$, analytic in $\rho$, which together with their first derivatives can be put in the asymptotic forms

$$
\begin{aligned}
u_{1} & =e^{\rho i x}\left(1+A /(\rho i)+O(1) / \rho^{2}\right), \\
u_{1}^{\prime} & =\rho i e^{\rho i x}\left(1+A /(\rho i)+O(1) / \rho^{2}\right), \\
u_{2} & =e^{-\rho i x}\left(1-A /(\rho i)+O(1) / \rho^{2}\right), \\
u_{2}^{\prime} & =-\rho i e^{-\rho i x}\left(1-A /(\rho i)+O(1) / \rho^{2}\right),
\end{aligned}
$$

valid $0 \leqq x \leqq 1, J(\rho) \geqq-c, c \geqq 0$. If $W\left(u_{1}, u_{2}\right)$ denotes the Wronskiun of the two functions $u_{1}$ and $u_{2}$, then the functions $v_{1}=-u_{2} / W, v_{2}=+u_{1} / W$ and their first derivatives have the asymptotic forms

$$
\begin{aligned}
v_{1} & =e^{-\rho i x}\left(1-A /(\rho i)+O(1) / \rho^{2}\right) /(2 \rho i), \\
v_{1}^{\prime} & =-e^{-\rho i x}\left(1-A /(\rho i)+O(1) / \rho^{2}\right) / 2, \\
v_{2} & =-e^{\rho i x}\left(1+A /(\rho i)+O(1) / \rho^{2}\right) /(2 \rho i), \\
v_{2}^{\prime} & =-e^{\rho i x}\left(1+A /(\rho i)+O(1) / \rho^{2}\right) / 2,
\end{aligned}
$$

valid on the same range.

The part of the lemma concerning the functions $u_{1}$ and $u_{2}$ is a special case of a general theorem due to Birkhoff. With the aid of Birkhoff's paper and a few comments upon it contained in a later one by the author, the truth of the statement is readily perceived.* The second part of the lemma, concerning the functions $v_{1}$ and $v_{2}$, is obtained by direct substitution.

* For the proofs see Birkhoff, these Transactions, vol. 9 (1908), pp 219-231, and S, \$I. The lemma is also a special case of a theorem of Tamarkin, $T_{2}$, Theorem 5. 
LEMMA II. If $\Gamma_{n}$ is a semicircle of radius $R_{n}$ with the origin as center, lying on the half-plane $J(\rho) \geqq 0$, then

$$
\int_{\Gamma_{n}}(O(1) / \rho) d \rho=O(1) .
$$

LEMMA III. If $0<a \leqq x \leqq b<1$, then

and

$$
\int_{\Gamma_{n}} \rho^{k} e^{\rho i x}\left(1-\rho^{2} / R_{n}^{2}\right)^{k+l} O(1) d \rho=O\left(R_{n}^{-l}\right), \quad k \geqq 0, l \geqq 0,
$$

$$
\int_{\Gamma_{n}} \rho^{k} e^{\rho i(1-x)}\left(1-\rho^{2} / R_{n}^{2}\right)^{k+l} O(1) d \rho=O\left(R_{n}^{-l}\right), \quad k \geqq 0, l \geqq 0 .
$$

Lemma IV. The integral $\int_{\Gamma_{n}} e^{\rho i} O(1) d \rho$ is $O(1)$.

LEммA V. If $0 \leqq \alpha \leqq x \leqq 1$, then

$$
\int_{\alpha}^{x} \int_{\Gamma_{n}}\left(e^{\rho i(x-y)} O(1) / \rho\right) d \rho d y=o(1)
$$

and, if $0 \leqq x \leqq \alpha \leqq 1$, then

$$
\int_{\alpha}^{x} \int_{\Gamma_{n}}\left(e^{\rho i(y-x)} O(1) / \rho\right) d \rho d y=o(1),
$$

uniformly on the ranges considered.

Lemmas II, III, V are in essence Lemmas III', $\mathrm{V}^{\prime}, \mathrm{VI}^{\prime}$ of our paper cited above as $\mathrm{S}$; we refer to those lemmas for proofs. Lemma IV is obviously little different from the first part of Lemma III, with $x=1$ and $k=l=0$.

LEMMA VI. If $\delta(\rho)$ is an analytic function with the asymptotic form

$$
\left[\theta_{1}\right] e^{2 \rho i}+\left[\theta_{0}\right] e^{\rho i}+\left[\theta_{2}\right], \quad \theta_{1} \theta_{2} \neq 0,
$$

on the region $J(\rho) \geqq-c$, then the equation $\delta=0$ has infinitely many roots in that region, distributed asymptotically near the roots of the equation

$$
\theta_{1} e^{2 \rho i}+\theta_{0} e^{\rho i}+\theta_{2}=0 .
$$

If these points are removed from the $\rho$-plane by deleting the interior of a small circle of preassigned radius described about each of them as center, the function $1 / \delta$ is $O(1)$ in the remaining portion of the region $J(\rho) \geqq-c$. The region obtained when $c=0$ we denote by $\Sigma^{\prime}$.*

${ }^{*} \mathrm{~B}_{1}$, p. 393; $\mathrm{B}_{2}$, pp. 120-121; $\mathrm{T}_{1}$, p. 353. The notation $[a]$ means as usual $a+O(1) / \rho$. 
LEMMA VII. If $\delta$ and $\bar{\delta}$ are functions of the type described in Lemma VI, with $\theta_{0}=\bar{\theta}_{0}, \theta_{1}=\bar{\theta}_{1}, \theta_{2}=\bar{\theta}_{2}$, then on $\Sigma^{\prime}$ we have $\delta / \bar{\delta}=[1]$. $^{*}$

LEMma VIII. If $F(x, y)$ is continuous for $x$ and $y$ on the interval $(a, b)$, then

$$
\int_{a}^{b} F(x, y) \sin R(x-y) d y=o(1)
$$

uniformly $a \leqq x \leqq b$ as $R$ becomes infinite. $\dagger$

We can approximate to $F$ by a function $F_{1}$ constant on each rectangle of a network fitted on the square $a \leqq x \leqq b, a \leqq y \leqq b$, so that

$$
\left|\int_{a}^{b}\left(F-F_{1}\right) \sin R(x-y) d y\right| \leqq \epsilon / 2 .
$$

Then for $R$ sufficiently great

Consequently,

$$
\left|\int_{a}^{b} F_{1} \sin R(x-y) d y\right| \leqq \epsilon / 2 .
$$

$$
\left|\int_{a}^{b} F \sin R(x-y) d y\right| \leqq \epsilon
$$

when $R$ is taken large enough, as we wished to show.

LEMMa IX. If we denote by $k$ a fixed positive integer; by $c_{i}, i=1, \cdots, k$, $a$ set of distinct constants, real or complex; and by $P_{i n}, Q_{i n}, i=1, \cdots, k$, a set of polynomials of degree less than or equal to $m$ independent of $n$, for $n=1$, $2,3, \cdots$, with real or complex coefficients-then a necessary and sufficient condition that as $n$ becomes infinite

$$
\sum_{i=1}^{k}\left(P_{i n} \cos \left(2 n \pi+c_{i}\right) x+Q_{i n} \sin \left(2 n \pi+c_{i}\right) x\right)=o(1)
$$

on a fixed interval $(a, b)$ is that the coefficients in the polynomials be o(1).

The sufficiency of the condition is obvious. To prove its necessity we let $s_{n}$ represent the sum of the absolute values of the coefficients in the polynomials $P$ and $Q$, and then derive a contradiction from the supposition that there can be found an infinite sequence of values of $n$ for which the quantities $s_{n}$ have a positive lower bound. If such a sequence exists, it is clear that we can determine a subsequence thereof for which

* S, Lemmas VIII and VIII'.

$\dagger$ For general results of this character, see Langer and Tamarkin, Bulletin of the American Mathematical Society, vol. 32 (1926), pp. 335-337. 


$$
P_{\text {in }} / s_{n}=P_{i}+o(1), \quad Q_{i n}=Q_{i}+o(1) \quad(i=1, \cdots, k) .
$$

On dividing the expression given in the statement of the lemma by $s_{n}$ we see that

$$
\sum_{i=1}^{k}\left(P_{i} \cos \left(2 n \pi+c_{i}\right) x+Q_{i} \sin \left(2 n \pi+c_{i}\right) x\right)=o(1)
$$

for the values of $n$ which we have retained. The real and imaginary parts of this equation can both be put in the form

$$
A(x) \cos 2 n \pi x+B(x) \sin 2 n \pi x=o(1)
$$

where $A^{2}+B^{2}$ is not identically zero in one of the two equations so obtained. By squaring the last equation and performing some simple trigonometric reductions it becomes

$$
A^{2}+B^{2}+\left(A^{2}-B^{2}\right) \cos 4 n \pi x+2 A B \sin 4 n \pi x=o(1) .
$$

Since $A$ and $B$ are continuous functions in view of their origin, the function on the left in this equation is bounded and its integral over $(a, b)$ approaches zero as $n$ becomes infinite; but, by the theorem of Riemann-Lebesgue, this integral has the limit $\int_{a}^{b}\left(A^{2}+B^{2}\right) d x$, which is different from zero unless $A^{2}+B^{2}$ vanishes identically. This gives us the desired contradiction; we must have $s_{n}=o(1)$ and can thus establish the lemma at once.

LEMMA X. Let $\left\{n_{p}\right\}$ be a sequence of integers such that $\sum_{p=1}^{\infty} p^{2} / \log n_{p}$ converges, $n_{p+1}$ is greater than $2 n_{p}$, and $n_{p} /\left(p \log n_{p}\right)$ becomes infinite with $p . *$ Then the function defined by the equations

$$
\begin{gathered}
\phi(0)=\phi(1)=0, \\
\phi(x)=\left(2 p^{2} n_{p} / \log n_{p}\right) \sin 2 n_{p} \pi x, \quad 1 /(p+1) \leqq x<1 / p(p=1,2, \cdots),
\end{gathered}
$$

is integrable in the sense of Denjoy on $(0,1)$; and, if $c$ is any constant,

$$
\begin{aligned}
& \int_{0}^{1} \phi(y) \sin \left(2 n_{q} \pi+c\right) y d y=\left(n_{q} / \log n_{q}\right)(1+O(1 / q))+O(1), \\
& \int_{0}^{1} \phi(y) \cos \left(2 n_{q} \pi+c\right) y d y=\left(-c n_{q} / \log n_{q}\right)(1+O(1 / q))+O(1), \\
& \int_{0}^{1} \phi(y) y \sin 2 n_{q} \pi y d y=\left(n_{q} /\left(q \log n_{q}\right)\right)(1+O(1 / q))+O(1), \\
& \int_{0}^{1} \phi(y) y \cos 2 n_{q} \pi y d y=O(1) .
\end{aligned}
$$

* We might, for instance, take $n_{p}$ as the integral part of exp $p^{4}$. It is clear that $n_{p+1}>2 n_{p}$ and that $\log n_{p}=p^{4}+O(1)$. The other properties follow immediately. 
The function $\phi(x)$ is integrable in the sense of Denjoy, and non-absolutely integrable in the sense of Riemann, since the series

$$
\sum_{p=1}^{\infty} \int_{1 /(p+1)}^{1 / p} \phi(y) d y=\sum_{p=1}^{\infty} p^{2} O(1) / \log n_{p}
$$

is convergent. The four integrals involving $\phi$ can be investigated by actually performing the integration in each case. We treat the first of them as typical, omitting the proofs for the other three. Writing $I_{p}$ for the contribution to the integral from the interval $(1 /(p+1), 1 / p)$ we find

$$
\begin{aligned}
I_{p}= & \int_{1 /(p+1)}^{1 / p} \phi(y) \sin \left(2 n_{q} \pi+c\right) y d y \\
= & \left(p^{2} / \log n_{p}\right) \int_{1 /(p+1)}^{1 / p}\left(-\cos \left(2\left(n_{p}+n_{q}\right) \pi+c\right) y\right. \\
& \left.\quad+\cos \left(2\left(n_{p}-n_{q}\right) \pi-c\right) y\right) d y .
\end{aligned}
$$

Thus, when $p$ and $q$ are different,

$$
\begin{aligned}
I_{p}=p^{2} n_{p} O(1) /\left(\left(\log n_{p}\right)\left(2\left(n_{p}+n_{q}\right) \pi+c\right)\right) \\
+p^{2} n_{p} O(1) /\left(\left(\log n_{p}\right)\left(2\left(n_{p}-n_{q}\right) \pi-c\right)\right) .
\end{aligned}
$$

From the requirement that $n_{p+1}>2 n_{p}$ it is apparent that

$$
n_{p} /\left(2\left(n_{p}+n_{q}\right) \pi+c\right)=O(1), n_{p} /\left(2\left(n_{p}-n_{q}\right) \pi-c\right)=O(1),
$$

whence $I_{p}=p^{2} O(1) / \log n_{p}, p \neq q$. When $p$ and $q$ are equal

$$
I_{q}=\left(q^{2} / \log n_{q}\right) O(1)+(\sin (c / q)-\sin (c /(q+1))) q^{2} n_{q} /\left(c \log n_{q}\right) .
$$

Now by Taylor's theorem with remainder we have

$$
(\sin (c / q)-\sin (c /(q+1))) / c=1 / q^{2}+O\left(1 / q^{3}\right) .
$$

In consequence,

$$
I_{q}=q^{2} / \log n_{q}+\left(n_{q} / \log n_{q}\right)(1+O(1 / q)) .
$$

Finally, the convergence of the infinite series whose general term is $p^{2} / \log n_{p}$ establishes the desired result

$$
\sum_{p=1}^{\infty} I_{p}=\left(n_{q} / \log n_{q}\right)(1+O(1 / q))+O(1) .
$$

The proof here is due to Titchmarsh.*

* Titchmarsh, Proceedings of the London Mathematical Society, (2), vol. 22 (1923-1924), pp. $x x v-x x v i$. 


\section{The EXPANSIONS OF SUMmable Functions}

We wish to investigate the formal expansions of a function integrable in the sense of Lebesgue on the interval $(0,1)$ in terms of the solutions of the differential system:

$$
\begin{aligned}
u^{\prime \prime}+\left(\rho^{2}+g\right) u=0, & 0 \leqq x \leqq 1, \\
W_{1}(u)=\alpha_{1} u^{\left(k_{1}\right)}(0)+\beta_{1} u^{\left(k_{1}\right)}(1)+\cdots=0, & \\
W_{2}(u)=\alpha_{2} u^{\left(k_{2}\right)}(0)+\beta_{2} u^{\left(k_{2}\right)}(1)+\cdots=0, & \\
& 2>k_{1} \geqq k_{2} \geqq 0,
\end{aligned}
$$

where $g(x)$ is summable on $(0,1)$, and the boundary conditions $W_{1}$ and $W_{2}$ are reduced to normal form and are regular.* There is defined by this differential system a Green's function $G\left(x, y ; \rho^{2}\right)$ meromorphic in the $\rho$-plane. The poles of $G$ on the region $\Im(\rho) \geqq-c$ occur at the roots of an equation of the form $\delta(\rho)=0$, where $\delta$ is of the character described in Lemma VI. On the region $\Sigma^{\prime}$ determined by the function $\delta$ we define a sequence of semicircles $\Gamma_{n}$ with centers at $\rho=0$ and radii $R_{n}$ such that the half-ring between $\Gamma_{n}$ and $\Gamma_{n+1}$ contains at least one pole of $G$ and as few others as possible. The numbers $R_{n}$ increase monotonely to $+\infty$. The formal expansion of a function $f(x)$ can then be expressed as

$$
\frac{1}{2 \pi i} \int_{0}^{1} f(y) \int_{\Gamma_{n}} 2 \rho G\left(x, y ; \rho^{2}\right) d \rho d y ;
$$

and means of order $l$ similar to the Riesz typical means of order $l$ for this expression can then be studied with the aid of the expression

$$
\frac{1}{2 \pi i} \int_{0}^{1} f(y) \int_{\Gamma_{n}} 2 \rho\left(1-\rho^{2} / R_{n}^{2}\right)^{l} G\left(x, y ; \rho^{2}\right) d \rho d y, l>0 .
$$

For fuller discussions of these facts we refer to other papers. $\dagger$

If we wish to examine simultaneously two differential systems of this type with Green's functions $G$ and $\bar{G}$, we can choose one sequence of semicircles $\Gamma_{n}$ lying on the region common to $\Sigma^{\prime}$ and $\bar{\Sigma}^{\prime}$, effective for both systems. In fact, the distribution of the poles of the two Green's functions, that is, of the roots of the two equations $\delta=0, \bar{\delta}=0$, is such that the semicircles are merely restricted to lie in a certain sequence of half-rings centered at the origin. This appears at once from Lemma VI.

* For the terms normal and regular, see $B_{1}$, pp. 382-383.

$\dagger \mathrm{B}_{1}$, pp. 373-380; S, §§II, III. 
If in Theorem XIX' of our paper cited as S we take $n=2$ and combine the results thus obtained for the two quadrants $0 \leqq \arg \rho \leqq \pi / 2, \pi / 2 \leqq$ $\arg \rho \leqq \pi$, we find

THEOREM I. If $f(x)$ is any function summable on the interval $(0,1)$, then the integral

$$
\int_{0}^{1} f(y) \int_{\Gamma_{n}}\left(2 \rho G-\left\{-i e^{\rho i(x-y)} ;-i e^{\rho i(y-x)}\right\}-\mathcal{D}(x, y ; \rho) / \theta_{2}\right) d \rho d y
$$

is o(1) uniformly $0 \leqq x \leqq 1$. The constant $\theta_{2}$ has the value

$$
i^{k_{1}+k_{2}}\left(\alpha_{1} \beta_{2}(-1)^{k_{2}}-\alpha_{2} \beta_{1}(-1)^{k_{1}}\right)
$$

and the function $\mathcal{D}$ the expression

$$
\left|\begin{array}{lll}
e^{\rho i x} & e^{\rho i(1-x)} & 0 \\
\alpha_{1} i^{k_{1}} & \beta_{1}(-i)^{k_{1}} & -\beta_{1} i^{k_{1}+1} e^{\rho i(1-y)}+\alpha_{1}(-i)^{k_{1}+1} e^{\rho i y} \\
\alpha_{2} i^{k_{2}} & \beta_{2}(-i)^{k_{2}} & -\beta_{2} i^{k_{2}+1} e^{\rho i(1-y)}+\alpha_{2}(-i)^{k_{2}+1} e^{\rho i y}
\end{array}\right|^{*}
$$

From this theorem we derive

THEOREM II. A necessary and sufficient condition that two differential systems of the second order with boundary conditions reduced to normal form and restricted to be regular be such that

$$
I_{n}=\frac{1}{2 \pi i} \int_{0}^{1} f(y) \int_{\Gamma_{n}} 2 \rho(G-\bar{G}) d \rho d y=o(1)
$$

uniformly $0 \leqq x \leqq 1$ for every summable function $f(x)$ is that the constants $\alpha$, $\beta, k$ of the boundary conditions satisfy one of the three relations

(1) $k_{1}=k_{2}=\bar{k}_{1}=\bar{k}_{2}=1$;

(2) $k_{1}=k_{2}=\bar{k}_{1}=\bar{k}_{2}=0$;

(3) $\quad k_{1}=\bar{k}_{1}=1, \quad k_{2}=\bar{k}_{2}=0, \quad \bar{\alpha}_{1}=\lambda \alpha_{1}, \quad \bar{\beta}_{1}=\lambda \beta_{1}, \quad \bar{\alpha}_{2}=\mu \alpha_{2}, \quad \bar{\beta}_{2}=\mu \beta_{2}$ where $\lambda$ and $\mu$ are constants different from zero.

By Theorem I,

$$
\begin{aligned}
2 \pi i I_{n}= & \int_{0}^{1} f(y) \int_{\Gamma_{n}}\left((\bar{A}-A) e^{\rho i(x+y)}+(\bar{B}-B) e^{\rho i(x+1-y)}\right. \\
& \left.+(\bar{C}-C) e^{\rho i(1-x+y)}+(\bar{D}-D) e^{\rho i(2-x-y)}\right) d \rho d y
\end{aligned}
$$

* See $\mathrm{S}$, Theorem XIX'. For the evaluation of the constant $\theta_{2}$, compare $\mathrm{B}_{1}, \mathrm{p} .383$. The notation $\{A ; B\}$ is employed to indicate a function reducing to $A$ when $x \geqq y$ and to $B$ when $x<y$. 
where the coefficients of the exponentials in the integral are constants depending only on the boundary conditions. By the theory of contour integration

$$
\int_{\Gamma_{n}} e^{\rho i \omega} d \rho=\int_{R_{n}}^{-R_{n}} e^{\rho i \omega} d \rho=-2 \sin \left(R_{n} \omega\right) / \omega,
$$

so that the explicit expression on the right of the equation above may be written

$$
\begin{gathered}
\int_{0}^{1} f(y) \phi(x, y ; n) d y, \\
\phi(x, y ; n)=2\left((A-\bar{A}) \psi_{n}(x+y)+(B-\bar{B}) \psi_{n}(1+x+y)\right. \\
\left.+(C-\bar{C}) \psi_{n}(1-x+y)+(D-\bar{D}) \psi_{n}(2-x-y)\right), \\
\psi_{n}(\omega)=\sin \left(R_{n} \omega\right) / \omega .
\end{gathered}
$$

A necessary and sufficient condition that $I_{n}$ be $o(1)$ uniformly $0 \leqq x \leqq 1$ is that $\int_{0}^{1} f(y) \phi(x, y ; n) d y=o(1)$ uniformly on the same range. By a well known theorem of Lebesgue a necessary condition that the last equation be true is that the function $\phi$ be uniformly bounded, $0 \leqq x \leqq 1,0 \leqq y \leqq 1 .^{*}$ Clearly this is impossible unless $A=\bar{A}, B=\bar{B}, C=\bar{C}, D=\bar{D}$; that is, unless $\phi$ is identically zero. Consequently these four equations constitute a necessary and sufficient condition that $I_{n}$ be $o(1)$ uniformly $0 \leqq x \leqq 1$. It can be shown algebraically that these equations imply one or another of the three sets of equations enumerated in the statement of the theorem and conversely. This establishes the theorem. $\dagger$

It is clear that the method employed could be extended to differential systems of arbitrary order with regular boundary conditions, since general theorems of which our Theorem I is a special case have been established for them. The algebraic difficulties would be considerable in the final stages of such a discussion.

\section{The EXPANSIONS FOR TOTALISABLE FUnCTIONS}

The formal expansion of a function integrable in the sense of Denjoy in terms of the solutions of a regular differential system of the second order is

* Lebesgue, Annales de la Faculté des Sciences de Toulouse, (3), vol. 1 (1909), pp. 52-55.

$\dagger$ The sufficiency of the conditions given by the theorem can be proved by some results of Tamarkin, $T_{2}$, pp. 104-112, and has been proved explicitly by the author, $S$, Theorem XVIII'. The necessity of the conditions can be deduced by the use of a theorem of Tamarkin concerning the uniform convergence of the Birkhoff series for a function of bounded variation, $T_{2}$, Theorem 14; the generalisation of the present theorem to Birkhoff series of arbitrary order could not be effected in that manner. 
given by the expressions of the preceding section. We shall restrict the differential equation $u^{\prime \prime}+\left(\rho^{2}+g\right) u=0$ by the requirement that $g(x)$ be an integral in the sense of Lebesgue, $0 \leqq x \leqq 1$. We first prove

THEOREM III. If $f(x)$ is totalisable, $0 \leqq x \leqq 1$, and if $\phi(x, y ; \rho)$ denotes the function

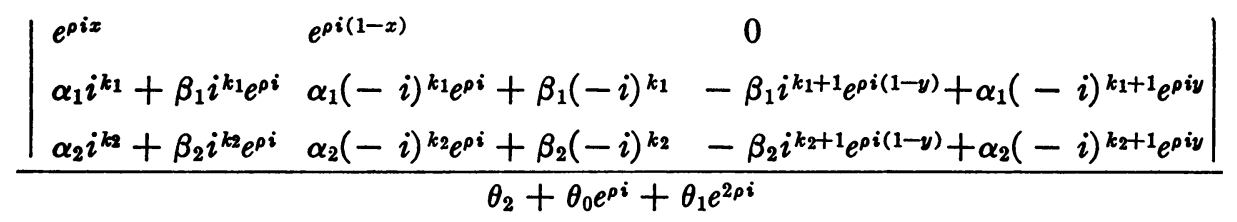

then the integral

$$
\begin{gathered}
\frac{1}{2 \pi i} \int_{0}^{1} f(y) \int_{\Gamma_{n}}\left(1-\rho^{2} / R_{n}^{2}\right)^{l}\left(2 \rho G-\left\{-i e^{\rho i(x-y)} ;-i e^{\rho i(y-x)}\right\}\right. \\
-\phi(x, y ; \rho)) d \rho d y
\end{gathered}
$$

is $o(1), l \geqq 0$, uniformly $0<a \leqq x \leqq b<1$.

The explicit formula for the Green's function is $G=\left\{u_{1}(x) v_{1}(y) ;-u_{2}(x) v_{2}(y)\right\}+\psi(x, y ; \rho)$, $\psi=\left|\begin{array}{lll}u_{1}(x) & u_{2}(x) & 0 \\ W_{1}\left(u_{1}\right) & W_{1}\left(u_{2}\right) & W_{11}\left(u_{1}\right) v_{1}(y)-W_{10}\left(u_{2}\right) v_{2}(y) \\ W_{2}\left(u_{1}\right) & W_{2}\left(u_{2}\right) & W_{21}\left(u_{1}\right) v_{1}(y)-W_{20}\left(u_{2}\right) v_{2}(y)\end{array}\right| \div\left|\begin{array}{ll}W_{1}\left(u_{1}\right) & W_{1}\left(u_{2}\right) \\ W_{2}\left(u_{1}\right) & W_{2}\left(u_{2}\right)\end{array}\right|$

where $u_{1}, u_{2}, v_{1}, v_{2}$ are the functions described in Lemma I, and $W_{k 0}$ and $W_{k 1}$ are the parts of the boundary condition $W_{k}$ involving $x=0$ and $x=1$ respectively.* We first study the integral

$$
\begin{aligned}
I_{n}=\int_{0}^{1} f(y) \int_{\Gamma_{n}}\left(1-\rho^{2} / R_{n}^{2}\right)^{l}\left(2 \rho\left\{u_{1}(x) v_{1}(y) ;-u_{2}(x) v_{2}(y)\right\}\right. \\
- \\
\left.-\left\{-i e^{\rho i(x-y)} ;-i e^{\rho i(y-x)}\right\}\right) d \rho d y .
\end{aligned}
$$

By the formula for integration by parts applicable to the integral with respect to $y$, we find

$$
\begin{array}{r}
I_{n}=F(1) \int_{\Gamma_{n}}\left(1-\rho^{2} / R_{n}^{2}\right)^{l}\left(i e^{\rho i(1-x)}-2 \rho u_{2}(x) v_{2}(1)\right) d \rho \\
-\int_{0}^{1} F(y) \int_{\Gamma_{n}} \chi(x, y ; \rho) d \rho d y,
\end{array}
$$

${ }^{*} B_{1}$, p. 378, p. 392; T1, p. 363. 
where

$$
\begin{aligned}
& F(x)=\int_{0}^{x} f d y, \\
& \begin{aligned}
\chi(x, y ; \rho)=\left(1-\rho^{2} / R_{n}^{2}\right)^{l}\left(2 \rho\left\{u_{1}(x) v_{1}^{\prime}(y) ;-u_{2}(x) v_{2}^{\prime}(y)\right\}\right. \\
\left.\quad-\rho\left\{-e^{\rho i(x-y)} ; e^{\rho i(y-x)}\right\}\right) .^{*}
\end{aligned}
\end{aligned}
$$

The first term on the right, when the asymptotic forms for $u_{2}$ and $v_{2}$ are substituted from Lemma $I$, is seen to be of the form

$$
\int_{\Gamma_{n}}\left(\left(1-\rho^{2} / R_{n}^{2}\right)^{l} e^{\rho i(1-x)} O(1) / \rho\right) d \rho=\int_{\Gamma_{n}} e^{\rho i(1-x)} O(1) d \rho / R_{n}
$$

and by Lemma III is $o(1)$ uniformly $0<a \leqq x \leqq b<1$. The second term becomes

$$
\begin{aligned}
\chi=(A(x)- & A(y)) i\left(1-\rho^{2} / R_{n}^{2}\right)^{l}\left\{e^{\rho i(x-y)} ; e^{\rho i(y-x)}\right\} \\
& +\left(1-\rho^{2} / R_{n}^{2}\right)^{l}\left\{e^{\rho i(x-y)} O(1) / \rho ; e^{\rho i(y-x)} O(1) / \rho\right\} .
\end{aligned}
$$

We denote the terms on the right by $\chi_{1}$ and $\chi_{2}$ respectively. Then

$$
\begin{aligned}
& \int_{0}^{1} F(y) \int_{\Gamma_{n}} \chi_{1} d \rho d y=\int_{0}^{1} F(y) \int_{R_{n}}^{-R_{n}} \chi_{1} d \rho d y \\
= & 2 \int_{0}^{R_{n}}\left(1-\rho^{2} / R_{n}^{2}\right)^{l} \int_{0}^{1} F(y)(A(x)-A(y)) \cos \rho(x-y) d y d \rho .
\end{aligned}
$$

This integral has the same behavior when $n$ becomes infinite as the integral with $l=0$, of which it is a Riesz sum. $\dagger$ The integral with $l=0$ is seen by Lemma VIII to be $o(1)$ uniformly, $0 \leqq x \leqq 1$; for, when the integration with respect to $\rho$ is performed, this integral can be written as

$$
2 \int_{0}^{1}(F(y)(A(x)-A(y)) /(x-y)) \sin R_{n}(x-y) d y .
$$

Next we discuss the integral involving $\chi_{2}$ by the aid of the theorem of Lebesgue cited in connection with Theorem II. Since $\chi_{2}=O(1) / \rho$ it follows from Lemma II that $\int_{\Gamma_{n}} \chi_{2} d \rho=O(1)$ uniformly, $0 \leqq x \leqq 1$; and from Lemma V

* The use of integration by parts is a standard device in the consideration of the Fourier series of totalisable functions. See for instance Nalli, Rendiconti del Circolo Matematico di Palermo, vol. 40 (1915), pp. 33-37, and Hobson, Proceedings of the London Mathematical Society, (2), vol. 22 (1924), pp. 420-424.

$\dagger$ Hardy and Riesz, The General Theory of Dirichlet Series (Cambridge Tracts, No. 18, 1915), Chapters IV and V. 
we see that $\int_{\alpha}^{\beta} \int_{\Gamma_{n}} \chi_{2} d \rho d y=o(1)$ uniformly, $0 \leqq x \leqq 1,0 \leqq \alpha \leqq \beta \leqq 1$. Lebesgue's theorem now yields the result $\int_{0}^{1} F(y) \int_{\Gamma_{n}} \chi_{2} d \rho d y=o(1)$ uniformly, $0 \leqq x \leqq 1$. Combining these results we see than $I_{n}$ is also $o(1)$ uniformly on the same range.

It remains for us to show that

$$
J_{n}=\int_{0}^{1} f(y) \int_{\mathrm{r}_{n}}\left(1-\rho^{2} / R_{n}^{2}\right)^{l}(\psi-\phi) d \rho d y=o(1)
$$

uniformly, $0<a \leqq x \leqq b<1$. An integration by parts yields the result

$$
\begin{gathered}
J_{n}=F(1) \int_{\Gamma_{n}}\left(1-\rho^{2} / R_{n}^{2}\right)^{l}(\psi(x, 1 ; \rho)-\phi(x, 1 ; \rho)) d \rho \\
-\int_{0}^{1} F(y) \int_{\Gamma_{n}}\left(1-\rho^{2} / R_{n}^{2}\right)^{l} \frac{\partial}{\partial y}(\psi-\phi) d \rho d y .
\end{gathered}
$$

If in the expressions $\psi-\phi,(\partial / \partial y)(\psi-\phi)$ we substitute the asymptotic expansions from Lemma $I$, expand the determinants involved, and evaluate the coefficients of the various exponentials occurring therein by the aid of Lemmas VI and VII, we find

$$
\begin{aligned}
\psi-\phi & =e^{\rho i x} O(1) / \rho+e^{\rho i(1-x)} O(1) / \rho, \\
\frac{\partial}{\partial y}(\psi-\phi) & =e^{\rho i x} O(1)+e^{\rho i(1-x)} O(1),
\end{aligned}
$$

for $0 \leqq x \leqq 1,0 \leqq y \leqq 1$, and for all $\rho$ on $\Sigma^{\prime} .^{*}$ Consequently,

$$
\int_{\Gamma_{n}}\left(1-\rho^{2} / R_{n}^{2}\right)^{l}(\psi-\phi) d \rho=\int_{\Gamma_{n}}\left(1-\rho^{2} / R_{n}^{2}\right)^{l}\left(e^{\rho i x} O(1)+e^{\rho i(1-x)} O(1)\right) d \rho / R_{n}
$$

is $o$ (1) uniformly, $0<a \leqq x \leqq b<1,0 \leqq y \leqq 1$, by Lemma III. We make twofold use of this fact. In the first place, it shows that the first term in $J_{n}$ is $o$ (1) uniformly on $(a, b)$. In the second place, it provides us with one of the two sufficient conditions of Lebesgue's theorem when we apply it to the second term in $J_{n}$; for it proves that

$$
\int_{\alpha}^{\beta} \int_{\mathrm{r}_{n}}\left(1-\rho^{2} / R_{n}^{2}\right)^{l} \frac{\partial}{\partial y}(\psi-\phi) d \rho d y
$$

is $o(1)$ uniformly on $(a, b)$. The other condition of that theorem is seen to be satisfied when we use Lemma III to prove that

* A somewhat more detailed discussion of a similar question is to be found in S, Theorem XV. 


$$
\int_{\Gamma_{n}}\left(1-\rho^{2} / R_{n}^{2}\right)^{l} \frac{\partial}{\partial y}(\psi-\phi) d \rho
$$

is $O$ (1) uniformly, $0<a \leqq x \leqq b<1,0 \leqq y \leqq 1$. Thus we see that the second term in $J_{n}$ is $o(1)$ uniformly on $(a, b)$.

Thus we have shown that $I_{n}+J_{n}$ is $o(1)$ uniformly on $(a, b)$; this is the assertion of the theorem.

Theorem IV. If $f(x)$ is totalisable, $0 \leqq x \leqq 1$, and if $l \geqq 1$, then

$$
\frac{1}{2 \pi i} \int_{0}^{1} f(y) \int_{\Gamma_{n}}\left(1-\rho^{2} / R_{n}^{2}\right)^{l}\left(2 \rho G\left(x, y ; \rho^{2}\right)-\left\{-i e^{\rho i(x-y)} ;-i e^{\rho i(y-x)}\right\}\right) d \rho d y
$$

is o(1) uniformly, $0<a \leqq x \leqq b<1$. Thus if $G$ and $\bar{G}$ are the Green's functions for two regular differential systems of the second order, and if the sequence of semicircles $\Gamma_{n}$ is chosen to apply to both, then

$$
\frac{1}{2 \pi i} \int_{0}^{1} f(y) \int_{\Gamma_{n}} 2 \rho\left(1-\rho^{2} / R_{n}^{2}\right)^{l}(G-\bar{G}) d \rho d y=o(1), \quad l \geqq 1,
$$

uniformly on the same range. In particular $\bar{G}$ may be chosen as the Green's function of the Fourier differential system

$$
u^{\prime \prime}+\rho^{2} u=0, \quad u(0)-u(1)=u^{\prime}(0)-u^{\prime}(1)=0 \text {; }
$$

consequently, the behavior of the sum of order $l \geqq 1$ for an arbitrary expansion of the class considered is the same as that of a sum of order $l$ for the corresponding Fourier series.

Since on $\Sigma^{\prime}$

$$
\begin{aligned}
1 /\left(\theta_{2}+\theta_{0} e^{\rho i}+\theta_{1} e^{2 \rho i}\right) & =1 / \theta_{2}-\left(\theta_{0} e^{\rho i}+\theta_{1} e^{2 \rho i}\right) /\left(\theta_{2}\left(\theta_{2}+\theta_{0} e^{\rho i}+\theta_{1} e^{2 \rho i}\right)\right) \\
& =1 / \theta_{2}+e^{\rho i} O(1),
\end{aligned}
$$

we can write

$$
\phi(x, y ; \rho)=A e^{\rho i(x+y)}+B e^{\rho i(x+1-y)}+C e^{\rho i(1-x+y)}+D e^{\rho i(2-x-y)}+e^{\rho i} O(1),
$$

where the coefficients of the exponentials are the constants defined in the course of the proof of Theorem II. In the same way

$$
\frac{\partial \phi}{\partial y}=A \rho i e^{\rho i(x+y)}-B \rho i e^{\rho i(x+1-y)}+C \rho i e^{\rho i(1-x+y)}-D \rho i e^{\rho i(2-x-y)}+\rho e^{\rho i} O(1) .
$$


Lemma III then shows that

$$
\begin{aligned}
& \int_{\Gamma_{n}}\left(1-\rho^{2} / R_{n}^{2}\right)^{l} \phi d \rho=o(1), \quad l \geqq 1, \\
& \int_{\Gamma_{n}}\left(1-\rho^{2} / R_{n}^{2}\right)^{l} \frac{\partial \phi}{\partial y} d \rho=O(1), \quad l \geqq 1,
\end{aligned}
$$

uniformly, $0<a \leqq x \leqq b<1,0 \leqq y \leqq 1$. From the theorem of Lebesgue which we have used so frequently we conclude that

$$
\begin{gathered}
\int_{0}^{1} f(y) \int_{\Gamma_{n}}\left(1-\rho^{2} / R_{n}^{2}\right)^{l} \phi d \rho d y=F(1) \int_{\Gamma_{n}}\left(1-\rho^{2} / R_{n}^{2}\right)^{l} \phi(x, 1 ; \rho) d \rho \\
-\int_{0}^{1} F(y) \int_{\Gamma_{n}}\left(1-\rho^{2} / R_{n}^{2}\right)^{l} \frac{\partial \phi}{\partial y} d \rho d y=o(1), \quad l \geqq 1,
\end{gathered}
$$

uniformly on $(a, b)$. By combining this result with Theorem III the present theorem is established without difficulty.

THEOREM V. If for an arbitrary function totalisable on $(0,1)$ and for some fixed value of $k$

$$
I_{n}=\frac{1}{2 \pi i} \int_{0}^{1} f(y)\left(\int_{\Gamma_{n}} 2 \rho G d \rho-\int_{\Gamma_{n+k}} 2 \rho \bar{G} d \rho\right) d y=o(1)
$$

uniformly or not on a fixed interval, however small, then it is necessary that the boundary conditions of the differential systems considered satisfy one of the relations (1)-(3) of Theorem II. Conversely, if the boundary conditions of the two systems are so related, then

$$
\frac{1}{2 \pi i} \int_{0}^{1} f(y) \int_{\Gamma_{n}} 2 \rho(G-\bar{G}) d \rho d y=o(1)
$$

uniformly, $0<a \leqq x \leqq b<1$.

As a consequence of Theorem III, it is sufficient to consider differential systems of the form

$$
\begin{aligned}
& u^{\prime \prime}+\rho^{2} u=0, \\
& \alpha_{1} u^{\left(k_{1}\right)}(0)+\beta_{1} u^{\left(k_{1}\right)}(1)=0, \\
& \alpha_{2} u^{\left(k_{2}\right)}(0)+\beta_{2} u^{\left(k_{2}\right)}(1)=0, \\
& 2>k_{1} \geqq k_{2} \geqq 0 .
\end{aligned}
$$

We shall evaluate $I_{n}$ explicitly in terms of the solutions of two such systems. 
There are three main cases to consider, according to the relations between $k_{1}$ and $k_{2}$ in the boundary conditions of such a system. In Case I, $k_{1}=k_{2}=1$, the boundary conditions can be put in the forn $u^{\prime}(0)=u^{\prime}(1)=0$, and the formal expansions are cosine series on $(0,1)$. In Case II, $k_{1}=k_{2}=0$, the expansions are sine series on $(0,1)$. In Case III, $k_{1}=1, k_{2}=0$, the expansions fall into three main types and two of these types are further subdivided into five sub-types each, as we shall now show.

In Case III we first compute the differential system adjoint to

$$
u^{\prime \prime}+\rho^{2} u=0, \quad \alpha_{1} u^{\prime}(0)+\beta_{1} u^{\prime}(1)=0, \quad \alpha_{2} u(0)+\beta_{2} u(1)=0,
$$

as

$$
v^{\prime \prime}+\rho^{2} v=0, \quad \beta_{2} v^{\prime}(0)+\alpha_{2} v^{\prime}(1)=0, \quad \beta_{1} v(0)+\alpha_{2} v(1)=0 .
$$

These systems are found to have solutions for $\rho=2 n \pi+a_{1}, \rho=2 n \pi+a_{2}$, where $\cos a_{1}=\cos a_{2}=-\left(\alpha_{1} \alpha_{2}+\beta_{1} \beta_{2}\right) /\left(\alpha_{1} \beta_{2}+\alpha_{2} \beta_{1}\right), 0 \leqq a_{1}<2 \pi, 0 \leqq a_{2}<2 \pi$. The condition that the boundary conditions be regular makes the denominator in this fraction different from zero. The three divisions of Case III are Type $1, a_{1} \neq a_{2}$; Type 2, $a_{1}=a_{2}=0$; Type $3, a_{1}=a_{2}=\pi$.

In Type 1,

$$
\begin{aligned}
u & =\alpha_{2} \sin \rho x-\beta_{2} \sin \rho(1-x) \\
& =\left(\alpha_{2}+\beta_{2} \cos \rho\right) \sin \rho x-\beta_{2} \sin \rho \cos \rho x \\
& =C_{1} \sin \rho x+C_{2} \cos \rho x, \\
v & =\beta_{1} \sin \rho x-\alpha_{1} \sin \rho(1-x) \\
& =\left(\beta_{1}+\alpha_{1} \cos \rho\right) \sin \rho x-\alpha_{1} \sin \rho \cos \rho x \\
& =D_{1} \sin \rho x+D_{2} \cos \rho x
\end{aligned}
$$

are the only solutions of the two systems corresponding to the same characteristic value $\rho$. For them

$$
\begin{aligned}
\int_{0}^{1} u v d x & =C_{1} D_{1} \int_{0}^{1} \sin ^{2} \rho x d x+\left(C_{1} D_{2}+C_{2} D_{1}\right) \int_{0}^{1} \sin \rho x \cos \rho x d x \\
& =C_{2} D_{2} \int_{0}^{1} \cos ^{2} \rho x d x=1 / K+O(1 / \rho)=1 / K+O(1 / n)
\end{aligned}
$$

where

$$
1 / K=\left(C_{1} D_{1}+C_{2} D_{2}\right) / 2=\left(\alpha_{1} \beta_{2}+\alpha_{2} \beta_{1}\right)\left(\sin ^{2} \rho\right) / 2 \neq 0 .
$$

Thus the formal expansion for a function $f(x)$ in terms of the functions $u$ is

$$
\sum_{(n)}(K+O(1 / n))\left(C_{1} \sin \rho x+C_{2} \cos \rho x\right) \int_{0}^{1} f(y)\left(D_{1} \sin \rho y+D_{2} \cos \rho y\right) d y
$$


where the sum is extended over the characteristic values of $\rho$ for which $\arg \rho$ is greater than or equal to 0 and less than $\pi$.

In Type $2, \cos \rho=1$ is the characteristic equation, so that we must have $\left(\alpha_{1}+\beta_{1}\right)\left(\alpha_{2}+\beta_{2}\right)=0$. The characteristic values $\rho=2 n \pi$ are double roots of the characteristic equation, so that the character of the expansions must be determined by computing the residues for the Green's function of the differential system. Since the general solution of the differential equation $u^{\prime \prime}+\rho^{2} u=0$ when $\rho=2 n \pi$ is a linear combination of $\cos 2 n \pi x$ and $\sin 2 n \pi x$ and satisfies the periodic boundary conditions $u^{\prime}(0)-u^{\prime}(1)=u(0)-u(1)=0$, the conditions

$$
\alpha_{1} u^{\prime}(0)+\beta_{1} u^{\prime}(1)=0, \alpha_{2} u(0)+\beta_{2} u(1)=0,\left(\alpha_{1}+\beta_{1}\right)\left(\alpha_{2}+\beta_{2}\right)=0
$$

must be compatible with them if $\rho=2 n \pi$ is to be a characteristic value for the differential system. Thus five cases are possible:

$$
\begin{aligned}
& u^{\prime}(0)-u^{\prime}(1)=u(0)-u(1)=0 ; \\
& u^{\prime}(0)-u^{\prime}(1)=u(0)=0 ; \\
& u^{\prime}(0)-u^{\prime}(1)=u(1)=0 ; \\
& u^{\prime}(0)=u(0)-u(1)=0 ; \\
& u^{\prime}(1)=u(0)-u(1)=0 .
\end{aligned}
$$

The residues of the Green's functions in the various cases can be computed directly, and are found to be

$$
\begin{aligned}
& 2 \cos 2 n \pi(x-y) ; \\
& 4(1-y) \sin 2 n \pi x \sin 2 n \pi y+4 x \cos 2 n \pi x \cos 2 n \pi y ; \\
& 4(1-x) \cos 2 n \pi x \cos 2 n \pi y+4 y \sin 2 n \pi x \sin 2 n \pi y ; \\
& 4(1-y) \cos 2 n \pi x \cos 2 n \pi y+4 x \sin 2 n \pi x \sin 2 n \pi y ; \\
& 4(1-x) \sin 2 n \pi x \sin 2 n \pi y+4 y \cos 2 n \pi x \cos 2 r \pi y .
\end{aligned}
$$

The series of Type 2 therefore fall into five subtypes.

The consideration of Type 3 where the characteristic equation is $\cos \rho$ $=-1$ is paralle: to that of Type 2 . The five sets of boundary conditions possible can be wbtained from those given under Type 2 by replacing each minus sign by a plus sign; and the corresponding residues of the Green's functions can be found by replacing $2 n$ by $2 n+1$ in the residues given under Type 2.

We are now prepared to discuss the consequences of the hypothesis that $I_{n}$ is $o(1)$ on a fixed interval. We form $I_{n}$ for the function $\phi$ of Lemma X; 
then we must have $I_{n+1}-I_{n}=o(1)$. If the two differential systems involved in $I_{n}$ have characteristic values of different forms, then $I_{n+1}-I_{n}$ is an algebraico-trigonometric sum of the type described in Lemma IX; and each system contributes a trigonometric function not contributed by the other. By Lemma $X$ there is at least one coefficient not $o(1)$ in this sum. Hence, according to Lemma IX, we arrive at the contradictory statement that $I_{n+1}-I_{n}$ cannot be $o(1)$. We may illustrate the discussion by considering two series under Case III, Type 1. We have

$$
\begin{aligned}
I_{n+1} & -I_{n} \\
= & \sum(K+O(1 / n))\left(C_{1} \sin \rho x+C_{2} \cos \rho x\right) \int_{0}^{1} \phi(y)\left(D_{1} \sin \rho y+D_{2} \cos \rho y\right) d y \\
& -\sum(\bar{K}+O(1 / n))\left(\bar{C}_{1} \sin \bar{\rho} x+\bar{C}_{2} \cos \bar{\rho} x\right) \int_{0}^{1} \phi(y)\left(\bar{D}_{1} \sin \bar{\rho} y+\bar{D}_{2} \cos \bar{\rho} y\right) d y
\end{aligned}
$$

where the first sum is extended over a limited number of values of $\rho$ of the form $\rho=2 n \pi+2 m_{1} \pi+a_{1}, \rho=2 n \pi+2 m_{2} \pi+a_{2}$, and the second over a limited number of values of $\bar{\rho}$ of similar form. We have also $\cos \rho \neq \cos \bar{\rho}$. The integrals in this sum are not $o(1)$ by Lemma X. Thus $I_{n+1}-I_{n}$ cannot be $o(1)$ in this case.

It remains for us to consider those cases in which the two systems have characteristic values of the same form. There are four of these: the comparison of a series in Case I with the corresponding series in Case II; the comparison of series of different subtypes in Case III, Type 2; the comparison of series of different subtypes in Case III, Type 3; and the comparison of series in Case III, Type 1.

When the two series compared are in Cases I and II respectively the reasoning given above applies without modification, since one is a cosine, the other a sine series.

When the two series are of different subtypes under Case III, Type 2, each series contributes to the expression $I_{n+1}-I_{n}$ an algebraico-trigonometric term not contributed by the other, and if the function for which the series are formed is taken as the function $\phi$ of Lemma $X$ at least one coefficient in $I_{n+1}-I_{n}$ is not $o(1)$. The desired result follows at once. For example, we consider series of subtypes (ii) and (iii). For the function $\phi$ the difference $I_{n+1}-I_{n}$ is seen to be

$$
\begin{aligned}
-4(1-2 x) \cos 2 n \pi x & \int_{0}^{1} \phi(y) \cos 2 n \pi y d y \\
& +4 \sin 2 n \pi x \int_{0}^{1} \phi(y)(1-2 y) \sin 2 n \pi y d y
\end{aligned}
$$


under the assumption that the integer $k$ introduced in the hypothesis of the theorem is zero. This expression clearly is not $o(1)$. If the integer $k$ were different from zero the same result would follow, since no function appearing in $I_{n+1}-I_{n}$ would come from both series.

When the two series are of different subtypes under Case III, Type 3, the reasoning of the preceding paragraph applies without change.

It remains for us to examine systems in Case III, Type 1, with the same characteristic values. Clearly the partial sums whose difference we denote by $I_{n}$ must be such that $I_{n+1}-I_{n}$ involves the same trigonometric functions from both sums; that is, the integer $k$ must be zero. Otherwise Lemmas IX and $\mathrm{X}$ would show that $I_{n+1}-I_{n}$, when formed for the function $\phi$, could not be $o(1)$. Hence we are able to write

$$
\begin{aligned}
& I_{n+1}-I_{n} \\
& =\sum(K+O(1 / n))\left(C_{1} \sin \rho x+C_{2} \cos \rho x\right) \int_{0}^{1} \phi(y)\left(D_{1} \sin \rho y+D_{2} \cos \rho y\right) d y \\
& \quad-\sum(\bar{K}+O(1 / n))\left(\bar{C}_{1} \sin \rho x+\bar{C}_{2} \cos \rho x\right) \int_{0}^{1} \phi(y)\left(\bar{D}_{1} \sin \rho y+\bar{D}_{2} \cos \rho y\right) d y
\end{aligned}
$$

where both sums are extended over the same set of values of $\rho$. On substituting the evaluations of the integrals as given in Lemma $\mathrm{X}$, we find, for values of $n$ in the sequence $\left\{n_{q}\right\}$ of that lemma,

$$
\begin{array}{r}
I_{n_{q}+1}-I_{n_{q}}=\sum\left(\left(\left(K C_{1} D_{1}-\bar{K} \bar{C}_{1} \bar{D}_{1}\right) n_{q} / \log n_{q}+o\left(n_{q} / \log n_{q}\right)\right) \sin \rho x\right. \\
\left.+\left(\left(K C_{2} D_{1}-\bar{K} \bar{C}_{2} \bar{D}_{1}\right) n_{q} / \log n_{q}+o\left(n_{q} / \log n_{q}\right)\right) \cos \rho x\right)
\end{array}
$$

where the sum is extended over a limited number of values of $\rho$ of the form $\rho=2 n \pi+2 m_{1} \pi+a_{1}, \rho=2 n \pi+2 m_{2} \pi+a_{2}$. This expression cannot be $o(1)$ unless the coefficients of the terms in $n_{q} / \log n_{q}$ vanish. Thus we find the following three necessary conditions:

$$
\cos \rho=\cos \bar{\rho}, K C_{1} D_{1}=\bar{K}_{1} \bar{D}_{1}, K C_{2} D_{1}=\bar{K}_{2} \bar{D}_{1} .
$$

These three equations can be expressed in terms of the constants of the boundary conditions and are found to imply the equations

$$
\bar{\alpha}_{1}=\lambda \alpha_{1}, \quad \bar{\beta}_{1}=\lambda \beta_{1}, \quad \bar{\alpha}_{2}=\mu \alpha_{2}, \quad \bar{\beta}_{2}=\mu \beta_{2},
$$

where $\lambda$ and $\mu$ are constants different from zero.

When all the results are collected, the necessary condition enunciated in the theorem is seen to summarize them.

In order to demonstrate the sufficiency of the condition, as asserted in the last part of the theorem, we need only notice that when the boundary 
conditions of the two systems fall into one of the three forms described the functions $\phi$ and $\phi$ of Theorem III are identical. It follows that

$$
\frac{1}{2 \pi i} \int_{0}^{1} f(y) \int_{\Gamma_{n}} 2 \rho(G-\bar{G}) d \rho d y=o(1)
$$

uniformly, $0<a \leqq x \leqq b<1$, by a direct application of Theorem III.

In closing we may note that Lemma IX and, therefore, Theorem $\mathrm{V}$ may be extended to the case where the expressions considered are $o(1)$, not on a fixed interval, but only on a fixed point set of positive Lebesgue measure. The proof of the necessary condition of Lemma IX requires modification only at the point where an integral over the interval $(a, b)$ is formed. Since Theorem $\mathrm{V}$ can be made just as general as the lemma on which it is based, the desired result is established.

Colomiria University, NEw YoRK, N. Y. 